\title{
Estimação do tamanho de população em um cultivo de tilápia (Oreochromis niloticus) via captura-marcação-recaptura
}

\author{
Eric Batista FERREIRA ${ }^{1}$ \\ Matheus Vilas Boas MILITANI ${ }^{2}$ \\ Vinícius Politi DUARTE ${ }^{3}$
}

\begin{abstract}
${ }^{1}$ Professor Adjunto II, Instituto de Ciências Exatas, Universidade Federal de Alfenas (Unifal-MG), Alfenas, MG, Brasil. E-mail: eric.ferreira@unifal-mg.edu.br.

${ }^{2}$ Graduando em Ciências Biológicas Licenciatura, Unifal-MG. E-mail: matheusmilitani@yahoo.com.br.

${ }^{3}$ Graduando em Ciências Biológicas Licenciatura, Unifal-MG. E-mail: viniciuspoliti@hotmail.com.
\end{abstract}

RESUMO: O método captura-marcação-recaptura é muito utilizado em áreas como Epidemiologia e Ecologia para estimar o tamanho de populações. O presente estudo teve como objetivo a estimação do tamanho da população de Tilápias-do-Nilo em um cultivo comercial, utilizando esse método. Resultados mostraram-se coerentes e denunciaram uma elevada mortalidade de peixes.

Palavras chave: Tilápia-do-Nilo, captura-marcação-recaptura, cultivo comercial.

\begin{abstract}
The method capture-mark-recapture is often used in areas such as Epidemiology and Ecology to estimate populations sizes. This study aimed at estimating the population size of Nile Tilapia, in a commercial growth, using such method. Results were consistent and reported a high mortality of fish.
\end{abstract}

Keywords: Nile Tilapia, capture-mark-recapture, commercial growth.

\section{INTRODUÇÃO}

Estudos sobre métodos de capturamarcação-recaptura (CMR) vêm sendo feitos desde 1783, iniciados por Laplace, para a estimação populacional na França. Em 1896, o pesquisador dinamarquês Carl G. J. Petersen foi o primeiro a empregar esse método no estudo de fluxo migratório de peixes no mar Báltico. Em 1930 Frederick Lincoln utilizou para estimar o tamanho da população de patos selvagens da América do Norte (JÚNIOR et al., 2010).

Desde então, métodos de CMR têm sido utilizado em várias áreas como na
Ecologia e Epidemiologia (ELKHOURY et al., 2007). Estudos vêm sendo feitos para estimar e monitorar o tamanho das populações de várias espécies, em populações fechadas e abertas. Este processo faz a estimativa correta de incidência e prevalência, ainda que os dados sejam fornecidos de forma incompleta.

Inicialmente tinha-se o intuito de estimar o tamanho total de uma população. Posteriormente, observou-se que seria possível dentro de uma população desconhecida ou de difícil conhecimento, estimar uma quantidade de uma subpopulação em particular, com características próprias (JÚNIOR et al., 2010). 
São várias as aplicações para esse método, dentre as quais encontra-se o estudo de populações incomuns ou esquivas, como usuários de drogas endovenosas. Além disso, o método de CMR tem sido utilizado para analisar o registro de Leishmaniose Visceral ou até mesmo o rendimento do professor em sala de aula. Há autores que propõem um modelo teórico para a implantação de um sistema de vigilância do diabetes mellitus na população idosa, baseado na utilização de fontes de morbidade e mortalidade usualmente disponíveis em nosso meio. Encontram-se também aplicações na estimação no número de defeitos distintos em inspeções de produtos, na dinâmica da frota de veículos estacionados no centro de determinadas cidades e foi usado para ajustar o censo dos Estados Unidos, de 1990 (WOLTER, 1991).

Coeli et al. (2000) citando White et al. (1982) afirmam que foi no campo da Ecologia, principalmente a partir das décadas de 1930 e 1940, onde se observou um consistente desenvolvimento teórico e aplicado do método de CMR para a estimação de populações de animais selvagens. Um exemplo da utilização do método foi no estudo da estimativa populacional de Parides anchises nephalion (Papilionidae) em uma área litorânea no Sudeste do Brasil realizado por Freitas e Ramos (2001).

$\mathrm{Na}$ ecologia, o método de CMR tem sido usado não somente para estimar populações de animais selvagens, mas também para determinar o movimento e as áreas de uso dos mesmos, como mostrado por Menezes Jr. (2008) e Campos et al. (2004), nos seus respectivos estudos sobre o deslocamento de Artibeus lituratus (Mammalia, Chiroptera) entre ilha e continente no Estado do Rio de Janeiro e o movimento e a área de uso do jacaré-dopantanal.

O método pode ser usado também para estimar a abundância, a razão sexual, o tempo máximo de residência a estrutura etária e outros parâmetros ecológicos de animais, como no estudo de adultos de $M$. themisto em duas praças públicas situadas na área central da cidade de Uberlândia, MG, como mostrado por Ruszczyk e Nascimento (1999).

Estudos envolvendo marcação e recaptura de peixes foi citado por Valentim et al. (2007) e realizado por Landa et al. (2001), a fim de observar o comportamento migratório de L. piscatorius e de L. budegassa.

No Brasil, um estudo para estimar o tamanho populacional e a sobrevivência de jovens tubarões-limão (Negaprion brevirostris) em uma área de berçário na Reserva Biológica do Atol das Rocas foi realizado por Freitas et al. (2009).

A aplicação do método para pesquisas em águas doces é bastante abundante como, por exemplo, no trabalho realizado por Hoeinghaus et al. (2003), que teve como objetivo avaliar a movimentação de Cichla no Rio Cinaruco, Venezuela.

Outra eficiência do método é estudar a capacidade de peixes migradores em localizar 
rotas alternativas na presença de um obstáculo Antônio et al. (2007) ou avaliar a passagem de peixes pelas escadas de barragens que foram construídas para o desvio de água para usinas (ALVES, 2007).

\section{CAPTURA-MARCAÇÃO-RECAPTURA}

Para o método de captura-marcaçãorecaptura, devem ser consideradas duas situações distintas: populações abertas e populações fechadas. Defini-se como população fechada, aquela em que não há mortes, nascimentos, migrações e emigração durante o estudo; diferentemente do conceito de população aberta em Ecologia, onde a diferença é a consideração dos efeitos de nascimento e de morte (JÚNIOR et al., 2010).

É necessário também que todos os elementos tenham a mesma probabilidade de captura (JÚNIOR et al., 2010).

Suponha, por exemplo, que se deseja estimar a população de peixes de uma determinada espécie em um lago (N) empregando a metodologia de capturamarcação-recaptura. Inicialmente seria capturada uma primeira amostra $\left(n_{1}\right)$ de peixes, que seriam contados, marcados e libertados. Após um período de tempo suficientemente longo para que os peixes marcados se misturassem aos não marcados, uma nova amostra $\left(n_{2}\right)$ seria capturada. Os peixes capturados na segunda amostra seriam contados e o número de peixes recapturados $\left(m_{2}\right)$ seria identificado. Assumindo-se a hipótese de independência entre as amostras, então se poderia concluir que a proporção de peixes recapturados em relação ao total de peixes capturados pela segunda amostra seria igual à proporção de peixes capturados pela primeira amostra em relação ao total da população.

Utilizando uma abordagem clássica, tem-se o estimador de Lincon-Petersen (HOOK e REGAL, 1995; IWGDMF, 1995), que foi utilizado por Laplace, sugerido por C. Petersen em 1986 e popularizado por F. Lincoln a partir de 1930:

$$
\frac{m_{2}}{n_{2}}=\frac{n_{1}}{N} \therefore N=\frac{n_{1} n_{2}}{m_{2}}
$$

em que $N$ é o tamanho da população; $n_{l}$ é o total de elementos capturados na primeira amostra; $n_{2}$ é o total de elementos capturados na segunda amostra; e $m_{2}$ é o número de elementos capturados na primeira e na segunda amostra.

Uma extensão do método de Petersen para a estimação da dimensão de uma população foi proposta por Schnabel (1938), para casos em que existe possibilidade de recolher mais do que duas amostras de diferentes dimensões, isto é, de dimensões $n_{l}$, $n_{2}, \ldots, n_{s}$. Neste método, tal como no método de Petersen, recolhe-se uma amostra inicial na qual marcam-se todos os $n_{1}$ indivíduos que são, em seguida, devolvidos à população; posteriormente, recolhe-se uma segunda 
amostra de dimensão $n_{2}$, registram-se os indivíduos marcados, cujo número se designa por $m_{2}$, e marcam-se novamente todos os indivíduos; o processo repete-se um determinado número $s$ de vezes. Para tal, torna-se necessário a utilização de marcas distintas em cada uma das capturas de forma a ser possível apurar em que altura os indivíduos foram recolhidos e, consequentemente, marcados.

Para melhor compreensão, considere a seguinte notação a seguir, que será utilizada ao longo da presente secção:

$N$ : dimensão total da população;

$s$ : número de amostras selecionadas (capturas);

$n_{i}$ : dimensão da amostra $i, \quad i=1,2, \mathrm{~K}, s$;

$m_{i}$ : número de indivíduos marcados na amostra $i$ de dimensão $n_{i}$.

$$
u_{i}=n_{i}-m_{i}
$$

$$
M_{i}=\sum_{j=1}^{i-1} u_{j}, \quad i=1,2, \mathrm{~K}, s+1 \text {, },: \text { número de }
$$

indivíduos imediatamente antes da amostra $i$ ter sido recolhida.

Chapman (1954) propôs um outro método para estimar a dimensão de uma população usando a captura e recaptura múltipla considera uma modificação do estimador de Petersen em cada uma das etapas realizadas. A importância dese método é não exigir que marcações distintas sejam feitas nos indivíduos a cada recaptura. Ele preconiza que, para cada uma das $s$ amostras de dimensão $n_{1}, n_{2}, \ldots, n_{s}$, respectivamente, o estimador modificado de Petersen de $N$ é dado pela equação (2):

$$
N_{i}=\frac{\left(M_{i}+1\right)\left(n_{i}+1\right)}{\left(m_{i}+1\right)}-1, i=2,3, \mathrm{~K}, s
$$

O estimador da variância de cada um dos valores obtidos pela equação (2) para a dimensão da população é dado pela equação (3):

$$
\operatorname{Mar}\left(N_{i}\right)=\frac{\left(M_{i}+1\right)\left(n_{i}+1\right)\left(M_{i}-m_{i}\right)\left(n_{i}-m_{i}\right)}{\left(m_{i}+1\right)^{2}\left(m_{i}+2\right)}
$$

A sugestão de Chapman (1954) para obter um estimador da dimensão de uma população fechada quando se utiliza mais do que uma recaptura, é considerar a média das diferentes estimativas obtidas pela equação (2), ou seja, considera-se o seguinte estimador:

$$
\bar{N}=\frac{\sum_{i=1}^{s} N_{i}}{s-1}
$$

cuja variância pode ser estimada por

$$
\operatorname{var}(\bar{N})=\frac{\sum_{i=2}^{s} \mathcal{N}_{i}}{(s-1)^{2}}
$$


Atendendo ao fato da distribuição de $\overline{\bar{N}} \mid$ ser assintoticamente Normal, considerase que o intervalo (aproximado) de confiança para $N$ é dado por:

$$
I C_{1-\alpha}(N)=\bar{N} \pm z_{\alpha / 2} \sqrt{\operatorname{Mgr}(\bar{N})}
$$

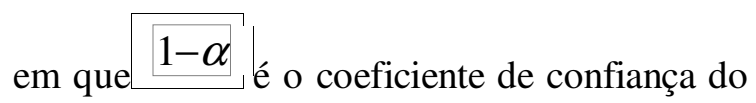

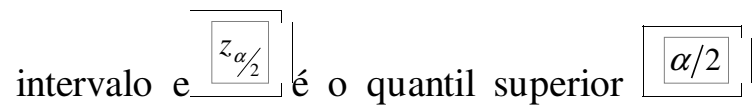
da distribuição Normal padrão.

Tilápia-do-Nilo (Oreochromis niloticus)

A Tilápia-do-Nilo (Figura 1) pertencente à família dos ciclídeos, é originária da bacia do rio Nilo, no Leste da África, encontrando-se amplamente disseminada nas regiões tropicais e subtropicais, como em Israel, no Sudeste Asiático (Indonésia, Filipinas e Formosa) e no Continente Americano (USA, México, Panamá e toda a América do Sul) (CARVALHO, 2006).

No Brasil foi introduzida em 1971, por intermédio do Departamento Nacional de Obras Contra a Seca (DNOCS) nos açudes do Nordeste, difundindo-se para todo o país (PROENÇA e BITTENCOURT, 1994; CASTAGNOLLI, 1992).

De acordo com diversos autores (CASTAGNOLLI，1992; KUBITZA，2000; GONZÁLEZ e QUEVEDO, 2001; ONO e
KUBITZA, 2003; CYRINO e CONTE, 2006) essa é uma espécie tropical cuja temperatura ideal para seu desenvolvimento varia entre 25 e $30^{\circ} \mathrm{C}$, tendo seu crescimento afetado abaixo de $15^{\circ} \mathrm{C}$ e não resistindo a temperaturas por volta de $9^{\circ} \mathrm{C}$.

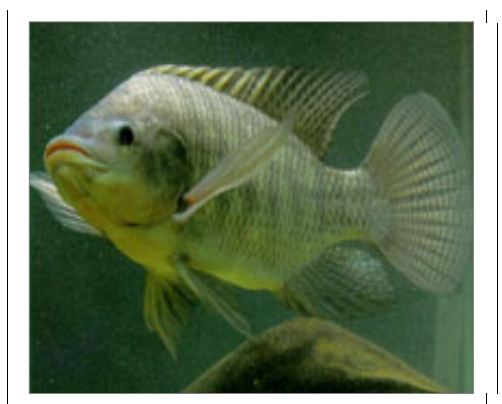

Figura 1 - Tilápia-do-Nilo Fonte: World Fish Center (2005).

A tilápia é um dos peixes com maior potencial para a aquicultura por diversas características (CASTAGNOLLI, 1992; ONO e KUBITZA, 2003; CYRINO e CONTE, 2006), como:

Aé precoce, de rápido crescimento, alimentando-se dos itens básicos da cadeia trófica e aceita grande variedade de alimentos;

A responde com a mesma eficiência a ingestão de proteínas de origem vegetal e animal;

A possui capacidade fisiológica de adaptarse em diferentes ambientes e sistemas de produção;

Aé resistente a doenças, densidades de estocagem elevadas e baixos teores de oxigênio dissolvido;

A apresenta carne saborosa com baixo teor de gordura $\left(0,9\right.$ g. $100 \mathrm{~g}^{-1}$ de carne $)$ e de calorias (117 kcal.100g ${ }^{-1}$ de carne), alto 
rendimento de filé (35 a 40\%) e ausência de espinhos em forma de "Y" (mioceptos), o que a torna apropriada para industrialização, e

A possui elevado valor comercial, principalmente nos países desenvolvidos.

\section{OBJETIVO}

Neste estudo teve-se como objetivo estimar o tamanho da população e a taxa de mortalidade de tilápias (Oreochromis niloticus) em um tanque fechado de cultivo comercial, por meio do método de CMR.

\section{METODOLOGIA}

As capturas das tilápias foram realizadas dia 19 de julho de 2011, em um tanque de cultivo comercial localizado em uma propriedade rural no município de Nepomuceno-MG.

A captura dos peixes, para posterior marcação em sua nadadeira caudal, foi através da utilização de uma tarrafa de 15 metros, malha $2,5 \mathrm{~mm}$. Foram feitas 26 capturas com um intervalo de 20 minutos entre elas.

$\mathrm{Na}$ primeira amostra os peixes foram marcados e libertados. A partir da segunda amostra os peixes recapturados foram identificados para uma posterior estimação da população.

As marcações das nadadeiras foram feitas com um furador de papéis promovendo furos de $0,6 \mathrm{~cm}$ de diâmetro nas nadadeiras caudais, a fim de não prejudicar a vida e nem a mobilidade dos indivíduos.

De posse dos dados, o método modificado de Petersen (descrito anteriormente) foi utilizado para estimar o tamanho populacional e a taxa de mortalidade do tanque.

\section{RESULTADOS E DISCUSSÃO}

Uma ilustração dos dados de captura e recaptura dos peixes pode ser observada na Figura 2. Nota-se que foram coletadas 26 amostras, das quais as capturas mantiveram-se basicamente variando entre 7 e 12 peixes e as recapturas variaram entre 0 e 5 . Observa-se também que nas recapturas houve um comportamento crescente do número de indivíduos coletados. Isso indica que o processo de CMR se portou como esperado.

Os dados foram tabulados e utilizados na equação (2) para estimar os $\sqrt{\mathscr{N}_{i}^{\circ}}$ de cada amostra. Com esses resultados foi calculada a sua variância, de acordo com a equação (3). De posse da variância, calculado o intervalo de confiança por meio da equação (6), equação essa que também utilizou-se do $\overline{\bar{N}}$ e sua variância, que foram obtidos por meio das equações (4) e (5), respectivamente.

Finalmente, estimou-se que o tamanho atual da população de tilápias no tanque analisado é de 360 indivíduos. Com 95\% de 
confiança, pode-se afirmar que o total populacional está entre 227 a 392 peixes.

De posse da estimativa do tamanho populacional e sabendo que inicialmente haviam 500 alevinos no tanque, pode-se inferir que a taxa de mortalidade dessa população foi de $28 \%$, conforme a equação (6).

$$
T M=\frac{P I-\bar{N}}{P I} \times 100
$$

em que $\stackrel{I M}{I M}$ é um estimador da taxa de mortalidade do tanque e $P I$ é a população inicial de alevinos.

O decréscimo populacional observado pode ter sido causado por diversas circunstâncias, por exemplo: predadores naturais (como lontras), dificuldades de estabelecimento na nova área, doenças oportunistas que podem ter acometido alguns alevinos na mudança de ambiente (criadouro/tanque), dentre outros.

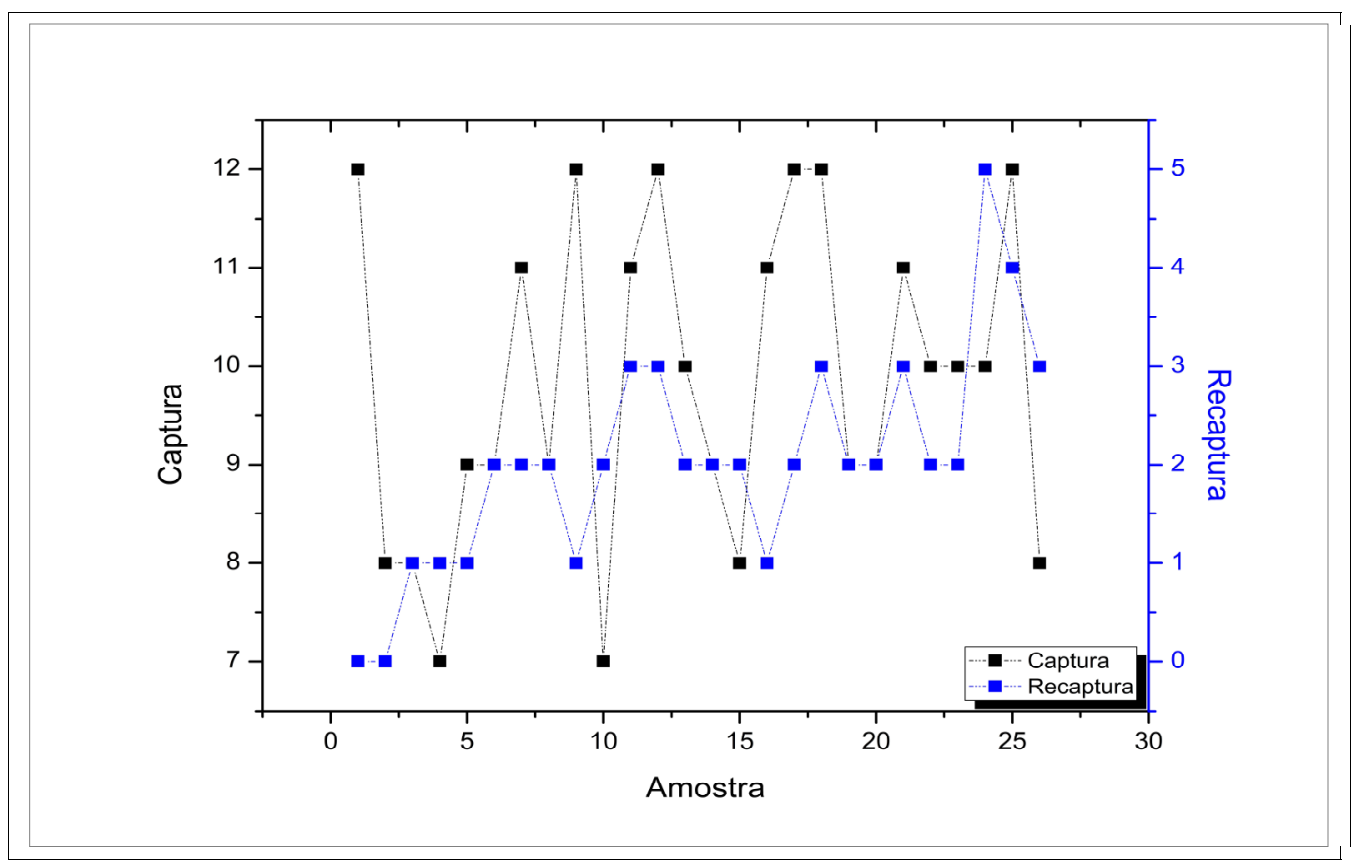

Figura 2 - Captura, marcação e recaptura dos peixes

Fonte: Próprios autores.

\section{CONCLUSÕES}

Conclui-se que a estimativa pontual (360) obtida para o tamanho da população em foco foi considerada coerente, pois o número inicial de alevinos colocados no tanque era 500 e, como não havia possibilidade de reprodução, a população atual deve ter tamanho menor ou igual a esse. Ainda, o intervalo de $95 \%$ de confiança obtido tem comprimento relativamente pequeno, o que o torna informativo; $\mathrm{e}$ apresenta limite superior menor que 500, o que também é desejado. 
Em termos práticos, observa-se uma diminuição considerável da população de tilápias nesse tanque, o que indica uma alta taxa de mortalidade/predação. Se nenhuma providência for tomada tal queda pode inviabilizar economicamente a criação comercial de peixes nesse tanque.

\section{REFERÊNCIAS BIOBLIOGRÁFICAS}

ALVES, C. B. M. Evaluation of fish passage through the Igarape Dam fish ladder (rio Paraopeba, Brazil), using marking and recapture. Neotropical Ichthyology. n. 5, p. 233-236. 2007.

ANTÔNIO, R. R.; AGOSTINHO, A. A.; PELICICE, F. M.; BAILLY, D.; OKADA, E. K.; DIAS, J. H. P. Blockage of migration routes by dam construction: can migratory fish find alternative routes? Neotropical Ichthyology, v. 5, n. 2, p. 177-184. 2007.

CAMPOS, Z.; MOURÃO, G.; COUTINHO, M.; MAGNUSSON, W. Movimento e área de uso do Jacaré-do-Pantanal. EMBRAPAPantanal. Boletim de Pesquisa 57. Corumbá, Brasil,. 33. 2004.

CARVALHO, E. D. Avaliação dos impactos da piscicultura em tanques-rede nas represas dos grandes tributários do alto Paraná (Tietê e Paranapanema): o pescado, a ictiofauna agregada e as condições limnológicas.

Relatório Científico (FAPESP). Botucatu, SP. 2006. 46p.

CASTAGNOLLI, N. Criação de peixes de água doce. Jaboticabal: FUNEP. 1992.189 p.

CHAPMAN, D. G. The Estimation of Biological Populations. Ann. Math. Statist. v. 25, p. 1-15. 1954.

COELI, C. M.; VERAS, R. P.; COUTINHO, E. S. F. Metodologia de captura-recaptura: uma opção para a vigilância das doenças não transmissíveis na população idosa. Cadastro de Saúde Pública, Rio de Janeiro, outubrodezembro, 2000.

CYRINO, J. E.; CONTE, L.; Tilapicultura em Gaiolas: produção e economia. In: José Eurico Possebon Cyrino e Elisabeth Criscuolo Urbinati (Eds.). AquaCiência 2004: Tópicos Especiais em Biologia Aquática e Aquicultura. Jaboticabal: Sociedade Brasileira de

Aqüicultura e Biologia Aquática, cap.12, p.151-171, 2006.

ELKHOURY, A. N. S. M.; CARMO, E. H.; SOUSA-GOMES, M. L.; MOTA, E. Analysis of visceral leishmaniasis reports by the capture-recapture method. Revista Saúde Pública, n. 41, v. 6, p. 1-6. 2007.

FREITAS, A. V. L.; RAMOS, R. R. Population biology of Parides anchises nephalion (Papilionidae) in a coastal site in Southeast Brazil. Brazilian Journal of Biology, v. 61, n. 4, p. 623-630. 2001.

FREITAS, R. H. A. de.; ROSA, R. S.; WETHERBEE, B. M.; GRUBER, S. H. Population size and survivorship for juvenile lemon sharks (Negaprion brevirostris) on their nursery grounds at a marine protected area in Brazil. Neotropical Ichthyology, v .7, n. 2, p. 205-212. 2009.

GONZÁLEZ, C. E.; QUEVEDO, E. T. Cultivo de las tilápias roja (Oreochromis spp.) y plateada (Oreochromis niloticus), cap.XIII. p. 283-299. In: GOMEZ, H.R.; DAZA, P.V.; AVILA, M.C.C. Fundamentos de Acuicultura Continental. Bogotá: Instituto Nacional de Pesca y Acuicultura, 2001, 423p.

HOEINGHAUS, D. J.; LAYMAN, C. A.; ARRINGTON, D. A.; WINEMILLER, K. O. Movement of Cichla species (Cichlidae) in a Venezuelan floodplain river. Neotropical Ichthyology, v. 1, n. 2, p. 121-126. 2003.

HOOK, E. B.; REAGAL, R. R. Capturerecapture methods in epidemiology: Methods and limitations. Epidemiologic Reviews, v. 17, p. 243-264. 1995. 
IWGDMF (International Working Group for Disease Monitoring and Forecasting).

Capture-recapture and multiple-record systems estimation: History and theoretical development. American Journal of

Epidemiology, 142:1047-1058. 1995.

JÚNIOR, E. L. S.; LYRA, B. S.; FERREIRA, C. M. L. Aplicação do método de capturarecaptura para estimar o rendimento do professor em sala de aula. In: SIMPÓSIO NACIONAL DE PROBABILIDADE E ESTATÍSTICA, 19, 2010, Campinas. Resumos... Campinas: Unicamp, 2010.

KUBITZA, F. Tilápia: Tecnologia e planejamento na produção comercial. $1^{\mathrm{a}} \mathrm{ed}$. Jundiaí: Fernando Kubitza, 2000. 289 p.

LANDA, J.; PEREDA, P.; DUARTE, R.; AZEVEDO, M. Growth of anglerfish (Lophius piscatorius and L. budegassa) in Atlantic Iberian waters. Fisheries Research, v. 51, p. 361-376. 2001.

MENEZES JR., L. F.; DUARTE, A. C.; NOVAES, R. L. M.; FAÇANHA, A. C.; PERACCHI, A. L.; COSTA, L. M.;

FERNANDES, A. F. P. D.; ESBÉRARD, C. E. L. Deslocamento de Artibeus lituratus (Olfers, 1818) (Mammalia, Chiroptera) entre ilha e continente no Estado do Rio de Janeiro, Brasil. Biota Neotropical. 2008, v. 8, n. 2, p. 243-245.

ONO, E. A.; KUBITZA, F. Cultivo de peixes em tanques-rede. $3^{\mathrm{a}}$ ed. Jundiaí: Eduardo A. Ono, 2003. 112p.

PROENÇA, C. E. M.; BITTENCOURT, P. R. L. Manual de Piscicultura Tropical. Brasília: IBAMA, 1994. 196 p.

RUSZCZYK, A.; NASCIMENTO, E. S., Biologia dos adultos de Methona themisto (Hübner, 1818) (Lepidoptera, Nymphalidae, Ithomiinae) em praças públicas de Uberlândia, Minas Gerais, Brasil. Revista Brasileira de Biologia. v. 59, p. 577-583. 1999. peixes do gênero Lophius (LOPHIIDAE, LOPHIIFORMES), com ênfase em Lophius gastrophysus. MIRANDA-RIBEIRO, 1915: STATUS ATUAL, Oecologia Brasiliensis, v. 11, n. 4, p. 503-520. 2007.

WHITE, G. C.; ANDERSON, D. R.; BURTHAM, K. P.; OTIS, D. L. CaptureRecapture and Removal Methods for Sampling Closed Populations. Los Alamos: Los Alamos National Laboratory. 1982.

WOLTER, K. M. Accouting for America's uncounted and miscounted. Science, v. 253, p. 12-5. 1991.

WORLD FISH CENTER. Oreochromis niloticus niloticus (Linnaeus, 1758) - Nile tilapia. 2005. Disponível em: http://www.fishbase.org/Photos/ThumbnailsS ummary.php?ID=2. Acesso em: 1 nov 2011.

VALENTIM, M. F. M.; VIANNA, M.;

CARAMASCHI, E. P. Biologia e ecologia de 